www.jmscr.igmpublication.org

Impact Factor (SJIF): 6.379

Index Copernicus Value: 71.58

ISSN (e)-2347-176x ISSN (p) 2455-0450

crossref DOI: https://dx.doi.org/10.18535/jmscr/v6i4.145

Journal Of Medical Science And Clinical Research

IGM Publication

An Official Publication of IGM Publication

\title{
Cytomorphological Spectrum of Ultrasound Guided Fine Needle Aspiration Cytology of Liver Lesions, and the Role of Cell Block Preparation and Immunohistochemistry in the Diagnosis: An Analysis of 638 Consecutive Aspirations
}

Authors

\section{Renu Sukumaran ${ }^{1}$, Nileena Nayak ${ }^{2}$, Jayasree Katoor ${ }^{3}$, Sindhu Nair $\mathbf{P}^{4}$, Anila KR ${ }^{5}$, Venugopal $\mathbf{M}^{6}$}

1,4,5 Assistant Professor, Division of Pathology, Regional Cancer Centre, Thiruvananthapuram, Kerala, India

${ }^{2}$ Additional Professor, Division of Pathology, Regional Cancer Centre, Thiruvananthapuram, Kerala, India

${ }^{3}$ Professor, Division of Pathology, Regional Cancer Centre, Thiruvananthapuram, Kerala, India

${ }^{6}$ Additional Professor, Division of Imageology, Regional Cancer Centre, Thiruvananthapuram, Kerala, India

Corresponding Author

Renu Sukumaran

Email: renu.sukumaran@gmail.com

\begin{abstract}
The differential diagnoses of space occupying lesions (SOL) of the liver are wide. It ranges from infectious and inflammatory processes to benign and malignant liver lesions which include primary and metastatic tumors. As the clinical, biochemical and imaging modalities have limitations in the accurate diagnosis, fine needle aspiration cytology places an important role in the categorization of liver SOL. The diagnostic difficulties encountered in FNAC are reduced by the use of cell block preparation and immunohistochemistry.

Aims and Objectives: To evaluate the spectrum of liver lesions, to study the cytological features of SOL of the liver and to evaluate the role of cell block preparation and immunohistochemistry in the diagnosis.

Materials and Methods: Retrospective review of the FNA slides of hepatic lesions during a period of three years from 2014 to 2016 was performed. The slides were studied for detailed cytological features and categorized into adequate, inadequate and inconclusive. The usefulness of cell block preparation and immunohistochemistry in the diagnosis were evaluated.

Results: A total of 638 liver aspirations were evaluated. 438 were adequate, 69 were inadequate and 131 were inconclusive. The age of the patients ranged from 0 to 88 years. Among the 438 adequate aspirations, 301 were metastatic. 250 cases were metastatic adenocarcinoma. Metastasis from squamous cell carcinoma, small cell carcinoma, neuroendocrine tumor, neuroblastoma, gastrointestinal stromal tumor and sarcoma were also encountered. There were 98 cases of hepatocellular carcinoma. Trabecular growth pattern, endothelial cuffing, macronucleoli, multinucleation and intranuclear inclusions were the most frequent findings in HCC. Cell block preparation was done in 107 cases. In nine cases, where the FNA was inconclusive, cell block preparation alone helped in making morphological diagnosis thus avoiding the need for a repeat procedure. IHC was done in cell block preparation of 66 cases which helped in the differentiation between well differentiated HCC from benign hepatic proliferations and poorly differentiated HCC from metastatic adenocarcinoma, to know the primary site of metastatic adenocarcinoma and to diagnose uncommon tumors.

Conclusion: In this study, we had a wide spectrum of liver lesions. The main diagnostic difficulties encountered were to differentiate well differentiated HCC from benign hepatic proliferations and poorly differentiated HCC from metastatic adenocarcinoma. Detailed cytological evaluation along with cell block preparation and subsequent IHC helped in the diagnosis.
\end{abstract}

Keywords: cell block, fine needle aspiration cytology, immunohistochemistry, liver lesions. 


\section{Introduction}

Guided FNA is very useful in the differential diagnosis of SOL of the liver. It can segregate different SOL will high degree of accuracy and can minimize further ancilliary investigations ${ }^{(1)}$. However diagnostic difficulties can occur in distinction of benign hepatocellular nodular lesions from reactive hepatocytes, distinction of well differentiated hepatocellular carcinoma from benign hepatocellular nodular lesions, distinction of poorly differentiated hepatocellular carcinoma from metastasis and in the determination of primary site of origin of malignant tumors ${ }^{(2)}$.

In certain situations, FNA may not yield sufficient information for an accurate diagnosis. The risk of false negative and indeterminate results are the major drawbacks of FNA. Sometimes there will be need for pattern recognition and need for ancillary studies like special stains and immunohistochemistry in rendering a precise diagnosis. In these circumstances, the preparation of cell block from residual material of the aspirate will give additional information thereby enhancing the diagnostic accuracy. FNA smears complemented by cell block will render an overall improvement in diagnosis.

\section{Materials and Methods}

In this retrospective study, 638 cases of liver aspirations over a period of three years from January 2014 to December 2016 were analyzed. Guided FNA was done using 20/21-gauge disposable spinal needle. Smears were immediately fixed in $95 \%$ methanol and papanicolaou stain was done. Cell blocks were prepared where there was excess material and IHC was done in required cases.

Depending on cell yield, the cases were classified as adequate, inadequate and inconclusive. In adequate aspirations, the cellularity was adequate and cell morphology was clear. Inadequate aspirations did not show epithelial cells. Cases with very low cellularity where the cell morphology was obscured due to inflammatory cells, necrosis, and blood or due to poor fixation and staining were classified as inconclusive.

The adequate aspirations were categorized into nonneoplastic and neoplastic. The neoplastic category was classified as benign and malignant. Malignant category was subdivided into primary and metastatic lesions. The distribution of each category was analyzed.

The morphologic findings in malignant liver lesions were studied in detail and the diagnostic clues and difficulties in differentiating various liver SOL were analyzed. The contribution of cell block preparation and IHC in rendering a definite diagnosis was studied.

\section{Results}

Of the 638 cases 438 were adequate, 69 were inadequate and 131 were inconclusive aspirations [Figure 1]. Age ranged from zero to 88 years.

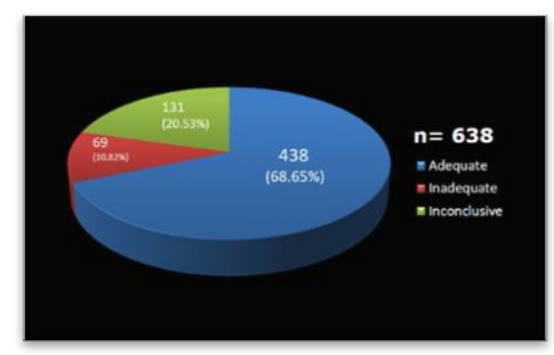

Figure 1: Categories of Aspirations

Among the 438 adequate aspirations, 11 cases were nonneoplastic lesions, 108 cases were primary hepatic malignancies, 301 cases were metastatic tumors, 4 were lymphoma and 14 were poorly differentiated carcinomas [Figure 2].

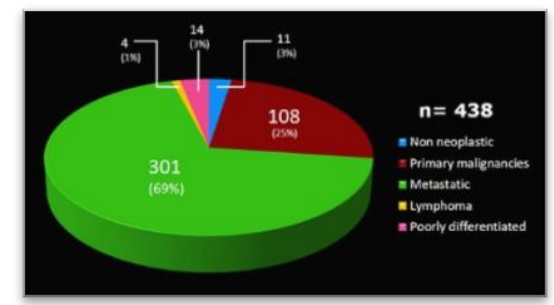

Figure 2: Distribution of diagnosis in Adequate aspirations

The nonneoplastic lesions included abscess (6 cases), granuloma (2 cases), cyst (2 cases) and fatty change (1 case).

Among malignant liver tumors 301 cases were categorized as metastatic lesions. 
Adenocarcinoma was the most common metastatic tumor which constituted 250 cases ( $83 \%$ of the metastatic tumors). This was followed by neuroendocrine tumor (15 cases), poorly differentiated carcinoma with neuroendocrine features (12 cases), small cell carcinoma (10 cases), squamous cell carcinoma (5 cases), neuroblastoma (4 cases), gastrointestinal stromal tumor ( 2 cases), sarcoma ( 2 cases) and epithelioid sarcoma ( 1 case). There were 4 cases of nonHodgkin lymphoma involving liver [Figure 3].

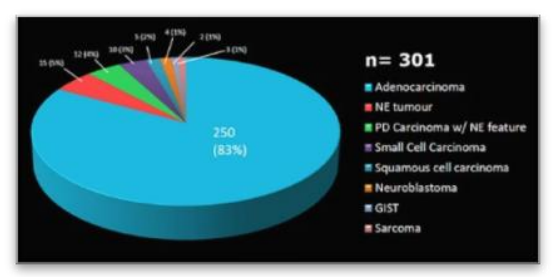

Figure 3: Distribution of Metastatic tumours Among the primary malignant tumors, hepatocellular carcinoma constituted 98 cases. Trabecular growth pattern, transgressing vessels, endothelial cuffing, macronucleoli, multinucleation and intranuclear inclusions were the most frequent findings in HCC. There were 8 cases of hepatoblastoma and 2 cases of cholangiocarcinoma.

Cell block preparation was done in 107 cases. In nine cases, where there was extremely low cellularity or loss of cell morphology due to obscuring factors or due to poor fixation and staining, the cell block preparations showed enough material to render a diagnosis. Immunohistochemical study was performed in 66 cases to differentiate well differentiated hepatocellular carcinoma from benign hepatic proliferations (2 cases), to differentiate poorly differentiated HCC from metastasis (31 cases), to assess the metastatic site (24 cases), in the evaluation of neuroendocrine tumors ( 7 cases) and to know the histogenesis of uncommon tumors (2 cases).

Fourteen cases were diagnosed as poorly differentiated carcinomas where the cell morphology, serum tumor marker values, and radiology findings were overlapping between primary and secondary tumors. In these cases, cell block preparation was not available for pattern recognition or for doing IHC studies.

\section{Discussion}

Space occupying lesions of the liver range from infectious and inflammatory processes to primary and secondary malignant lesions. Clinical, serological and radiological findings help to narrow down the differential diagnosis but do not always allow precise categorization of the lesions $(2,3)$.

Although markedly elevated serum AFP values give clue to the neoplastic nature of liver SOL, it is not a very good screening test. Normal serum AFP values does not exclude the possibility of hepatocellular carcinoma and elevated AFP levels can be seen in inflammatory conditions of liver.

Radiological findings help in the diagnosis but there is some overlap between different lesions ${ }^{(3)}$. Inflammatory conditions and diffuse liver diseases may mimic mass like lesions or appear as nonhomogenous lesions on imaging. Cystic change occurring in neoplastic lesions can mimic inflammatory and infectious conditions.

Fine needle aspiration cytology is a simple and cost effective diagnostic tool which will help in the precise diagnosis of various SOL of the liver and thereby minimize the need for further ancillary investigations.

In certain situations, FNA will not give sufficient information to render an accurate diagnosis and there is a chance of false negative and indeterminate results. This may be due to decreased cellularity (quantitative problems) or may be due to the lack of good morphological details (qualitative problems). The morphological details can be obscured by inflammatory cells, necrosis, and blood. Drying and crushing artifacts can affect the interpretation of cell morphology. The precise diagnosis of poorly differentiated and undifferentiated tumors can be challenging in cytology materials. Cell block preparation obtained from residual material of the aspirate will give additional information and thus enhance the diagnostic accuracy ${ }^{(4)}$. 
Hepatocellular carcinoma is the most common primary malignancy of the liver in adults. Liver is one of the most common sites for metastatic disease, accounting for $25 \%$ of all metastasis to

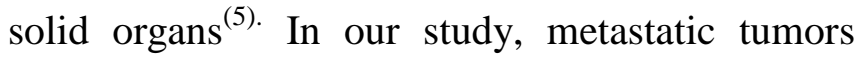
outnumbered primary liver tumors. There were 301 metastatic tumors and 108 primary malignancies. Majority of other studies also observed secondary tumors as the most common malignant liver lesions. In our study, $69 \%$ of hepatic lesions were metastatic tumors. This is in concordance with other studies where Barbhuiya et al observed $74.9 \%$ lesions, Rasania et al noted $70.4 \%$ lesions and Ali SR noted $58 \%$ of lesions were metastatic tumors ${ }^{(6,7,8)}$.

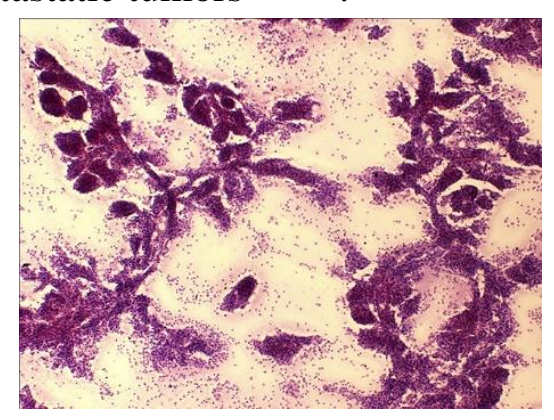

Figure 4: Cellular smear showing atypical cells in trabecular pattern (Pap,100x)

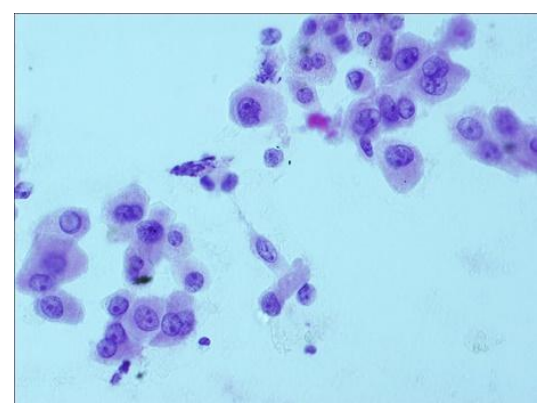

Figure 5: Atypical cells with abundant cytoplasm, prominent nucleoli and intranuclear inclusions (Pap, 400x)

The cytological diagnosis of hepatocellular carcinoma depends on the assessment on architectural features and cell morphology. Challenges in the diagnosis include separation of well differentiated HCC from benign hepatic proliferations and separation of poorly differentiated HCC from metastatic tumors ${ }^{(9)}$. Highly cellular smears showing cells arranged in broad trabeculae, monolayered sheets and pseudoacinar pattern with transgressing vessels and endothelial wrapping is highly suggestive of HCC [Figure 4]. Similar to the studies by Cohen et al and Green et al we also found trabecular pattern is the most common and most helpful pattern in recognizing $\mathrm{HCC}^{(10,11)}$. Transgressing vessels were noted in majority of cases of HCC but the feature of endothelial wrapping around the tumor cells were more specific in differentiating HCC from benign hepatic proliferations and from metastatic tumors. Although cell cohesion is the rule, singly dispersed malignant cells and atypical naked nuclei were common in HCC. Hepatocytic characteristics include polygonal cells with abundant granular cytoplasm, centrally placed round nuclei and prominent nucleoli. Multinucleation, multiple prominent eosinophilic nucleoli, intracytoplasmic and intranuclear inclusions were also noted in HCC [Figure 5]. Cellularity, broad trabeculea with transgressing vessels and endothelial wrapping, monotonous cell population and increased N/C ratio and absence of bile duct epithelium helped in the differentiation of well differentiated HCC from benign hepatic proliferations ${ }^{(2,9,12)}$. In poorly differentiated HCC search for the hepatocytic features along with correlation with clinical features, serum tumor marker values and imaging findings helped to arrive at a definite diagnosis.

In certain cases where the cytological findings were not sufficient to arrive at a definite diagnosis, cell block preparation helped to solve the diagnostic dilemma by providing the architectural details and providing additional material to do immunohistochemical studies. In cases of extremely well differentiated HCC, CD34 immunostain helped in assessing the vascular pattern [Figure 6]. In cases of poorly differentiated carcinoma, where cell block preparation provided enough material, an array of immunomarkers helped to differentiate poorly differentiated HCC from metastatic tumors and to identify the primary site in cases of metastasis. 


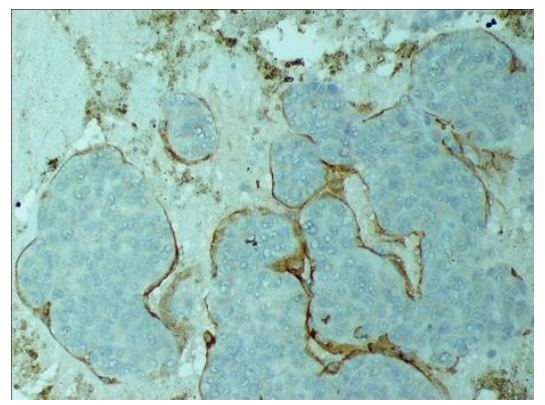

Figure 6: CD34 stain done in cell block preparation highlighting the vasculature (IHC,400x)

Liver is a common target organ for metastasis ${ }^{(5,6)}$. Multiple liver lesions of similar size will favor metastasis whereas single large lesion with or without satellite nodules in a background of cirrhosis will favor HCC. When dealing with liver FNA, one must be fully aware of any past history of malignancies, the tumor marker values and imaging findings. The presence of columnar cells, mucin, necrosis and inflammatory cells will give clue to the metastatic nature of the disease.

Highly cellular smears with columnar or cuboidal cells arranged in three dimensional clusters, acini, glandular pattern with increased N/C ratio, centrally/eccentrically placed nuclei, vacuolated pale cytoplasm will favor metastatic adenocarcinoma $^{(5,6,8)}$. Signet ring cells point to the gastric origin whereas adenocarcinoma with squamous differentiation will clue to the pancreatic origin. Extensive necrosis with columnar cells with nuclear palisading suggest colonic primary.

In our study, the adenocarcinoma was the most common metastatic tumor. It constituted $83 \%$ of secondary tumors. Common sites of primary tumors were breast, stomach, colon, pancreaticobiliary tract and lung. In cases with poorly differentiated cells, IHC done on cell block sections helped in pointing the primary site.

There were 15 cases of neuroendocrine tumors. Monotonous population of plasmacytoid cells without any nuclear pleomorphism, mitosis or necrosis gave clue to the diagnosis. Most cases the primary lesions were in the gastrointestinal tract and lung. In cases with diagnostic difficulty IHC studies with cytokeratin, synaptophysin, chromogranin and MIB1 labelling index helped in rendering the diagnosis [Figure 7].

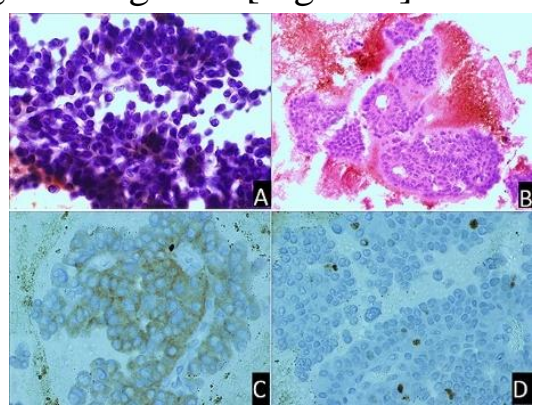

Figure 7: A) Cytology of Neuroendocrine tumor showing monotonous population of cells (Pap, 400x), B) Cell block preparation (H\&E,200x) C) Cells showing synaptophysin positivity (IHC,400x), D) Low MIB 1 labelling index (IHC,400x)

Cellular smears with small cells, scanty cytoplasm, finely granular chromatin, inconspicuous nucleoli, with nuclear moulding, smearing artifact, increased mitosis and necrosis were diagnostic of small cell carcinoma. Cases where these conclusive findings were lacking, IHC helped in diagnosis. Most common primary was in the lung followed by gastrointestinal tract.

The diagnosis of squamous cell carcinoma was made by the presence of atypical keratinized cells with hyperchromatic nuclei and tadpole shaped cells. Primaries were from the oral cavity and lung.

There were two cases of GIST metastasizing to liver, one confirmed by doing C KIT immunohistochemistry [Figure 8]. Three cases of sarcoma metastasis including one case of epithelioid sarcoma were also encountered in the study. In these cases, the awareness of previous history of sarcoma and the correlation with the initial biopsies helped in diagnosis.

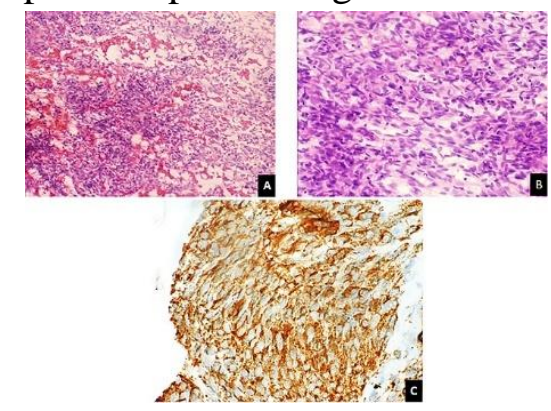

Figure 8: A) Cytology of gastrointestinal stromal tumor showing spindly cells (Pap, 400x), B) Cell 
block preparation $(\mathrm{H} \& \mathrm{E}, 400 \mathrm{x}), \mathrm{C})$ tumor cells showing C KIT positivity (IHC,400x).

Cell block preparation along with routine cytology smears will give additional information and will provide material for doing ancillary studies ${ }^{(13)}$. The use of cell block preparation can avoid further invasive procedures in certain situations where more amount of tissue is needed for ancillary studies. In the current study, cell block preparation was done in 107 cases. In nine cases where the material in cytology smears were scanty or inconclusive due to cell obscuring factors; cell block preparation provided adequate material for diagnosis. A more important role of cell block is in providing ample number of sections for IHC studies which will help to narrow down the differential diagnosis. As the commonest malignancy in liver is metastatic adenocarcinoma, the judicious use of IHC will help to point the primary site.

In our series, there were 14 cases which were diagnosed as poorly differentiated carcinoma. By morphology it was not possible to diagnose as primary or metastasis in these cases. We have done cell block preparation in 107 cases. If cell blocks are available in more number of cases, the inadequate and inconclusive categories should have less number of cases. Similarly, the poorly differentiated carcinoma could have been categorized as primary or metastatic using IHC panel.

\section{Conclusion}

Fine needle aspiration cytology is a very useful diagnostic tool in diagnosing various SOL of the liver with high degree of accuracy. Detailed study of morphological details along with correlation with clinical and radiological features will help in the diagnosis. In areas with diagnostic dilemma, the use of cell block and immunohistochemistry will help in the diagnosis. It is recommended to do cell block preparation in all possible cases to improve the diagnostic accuracy.

\section{References}

1. Ramdas A, Chopra R. Diagnostic accuracy of fine needle aspiration cytology of liver lesions. J Cytol2003;20:121-3.

2. Swamy M, Arathi C, Kodandaswamy C . Value of ultrasonography-guided fine needle aspiration cytology in the investigative sequence of hepatic lesions with an emphasis on hepatocellular carcinoma. J Cytol2011;28:178-84

3. Shah A, Jain GM. Fine needle aspiration cytology of the liver- A study of 518 cases.JCytol 2002;19:139-43.

4. 4.Coyhan K, Kupana SA, Bektas M, Cobar S, Tuzun A, CinarK, etal.The diagnostic value of on-site cytomorphological evaluation and cell block preparation in fine needle aspiration cytology of liver masses. Cytopathology 2006;17:267-74.

5. Ahuja A, Gupta N, Srinivasan R, Kalra N, Chawla Y, Rajwanshi A. Differentiation of hepatocellular carcinoma from metastatic carcinoma of the liver - clinical and cytological features. J Cytol2007;24:125-9

6. Barbhuiya M, Bhunia S, Kakkar M, Shrivastava B, Tiwari PK, Gupta S. Fine needle aspiration cytology of lesions of liver and gall bladder: An analysis of 400 consecutive aspirations. J Cytol 2014;31: 20-4.

7. Rasania A, Pandey CL, Joshi N. Evaluation of FNAC in diagnosis of hepatic lesion. J Cytol2007;24:51-4.

8. Ali SR, Jayabackthan L, Rahim S, Sharel MB, Prasad K, Hegdekatte N. Role of fine needle aspiration cytology in the diagnosis of hepatic lesions. Muller J Med Sci Res 2015;6:125-8

9. Wee A, Nilsson B. Highly well differentiated hepatocellular carcinoma and benign hepatocellular lesions. Can they be distinguished on fine needle aspiration biopsy? ActaCytol2003;47:1626. 
10. Cohen MB, Haber MM, Holly EA, Ahn DK, Bottles K, Stoloff AC. Cytologic criteria to distinguish hepatocellular carcinoma from nonneoplastic liver. Am J ClinPathol 1991; 95: 125-30.

11. Greene CA, Suen KC. Some cytologic features of hepatocellular carcinoma as seen in fine needle aspirates. ActaCytol 1984; 28: 713-8.

12. Soyuer I, Ekinci C, Kaya M, Genc Y, Bahar K. Diagnosis of hepatocellular carcinoma by fine needle aspiration cytology. Cellular features. Acta Cytol 2003;47:581-9.

13. Sheefa H, Lata J, Basharat M, Rumana M, Veena M, Utility of FNAC in conjunction with cell block for diagnosing spaceoccupying lesion (SOL) of liver with emphasis on differentiating hepatocellular carcinoma from metastatic SOL: Analysis of 61 cases. Oman Med J 2016;31:135-41. 